\title{
Aprendizaje de los profesores sobre alfabetización y métodos de enseñanza1
}

\author{
Teachers' Learning on Literacy and Teaching Methods
}

\section{Aprendizagem dos professores sobre alfabetização e métodos de ensino}

Rosa Julia Guzmán Rodríguez²

\section{Resumen}

Este artículo, producto de una investigación cualitativa adelantada en la Universidad de La Sabana con participación de diferentes grupos de profesores, contribuye a responder a la pregunta: ¿cómo aprenden los profesores a facilitar la alfabetización de sus estudiantes? Se buscaba identificar las relaciones entre teoría y práctica en la construcción de su conocimiento. Los resultados señalan que el conocimiento de los métodos de enseñanza de la lectura y la escritura se dan mediante la práctica, no responde a las necesidades de los niños y la evaluación que hacen a los estudiantes lleva a definir su situación, como problemas de aprendizaje.

\section{Palabras clave}

aprendizaje de los profesores; lectura; escritura; métodos de enseñanza

\section{Abstract}

This article is the result of a qualitative research performed at the Universidad de la Sabana in Colombia, in which many different groups of teachers participated. The paper helps to answer the research question "How do teachers learn to facilitate their students' literacy?" The purpose was to identify the relationship between theory and practice in knowledge construction. Results show that the understanding of teaching methods for reading and writing skills is given through practice, that it doesn't suffice children's learning needs, and that the evaluation that teachers have for their students rates them as learning issues.

\section{Keywords}

teachers' learning; reading; writing; teaching methods

\section{Resumo}

Este artigo, produto de uma pesquisa qualitativa realizada na Universidade de La Sabana com a participação de diversos grupos de professores, contribui à resposta da questão: como aprendem os professores a facilitar a alfabetização dos seus alunos? 0 objetivo era identificar as relações entre teoria e prática na construção de seu conhecimento. Os resultados indicam que o conhecimento dos métodos de ensino da leitura e a escrita é adquirido mediante a prática, não responde às necessidades das crianças e a avaliação dos estudantes define sua situação, como problemas na aprendizagem.

\section{Palavras chave}

aprendizagem dos professores; leitura; escrita; métodos de ensino

Artículo recibido el 25 de julio del 2016 y aprobado el 21 de diciembre del 2016

1 Este artículo es producto de la investigación Cómo aprenden los educadores del nivel inicial a diseñar estrategias que faciliten la alfabetización inicial en la línea de Pedagogía e Infancia, que hace parte del grupo de investigación Educación y educadores de la Universidad de La Sabana. Fue financiado por la Dirección de Investigación de la Universidad de La Sabana, Colombia (2008-2010), código EDu-22-2008.

2 Maestría en Pedagogía de la Universidad de La Sabana, Chía, Colombia. Correo electrónico: rosa.guzman@unisabana.edu.co 


\section{Introducción}

Para la Facultad de Educación de la Universidad de La Sabana es muy importante investigar sobre la formación de los profesores. En una investigación cuya pregunta fue ¿cómo aprenden los educadores del nivel inicial a diseñar estrategias que faciliten la alfabetización inicial?, se planteó como una de las categorías de análisis los métodos de enseñanza de la lectura y la escritura, dada la resonancia que este asunto tiene en el ámbito educativo, y las implicaciones que de las ideas que tengan los educadores con respecto a dichos métodos y del uso de uno $\mathrm{u}$ otro se derivan para el aprendizaje de los niños ${ }^{3}$, mediado por las prácticas cotidianas en las aulas. Se trabajó con un enfoque de investigación cualitativa y su alcance fue descriptivo exploratorio.

En este artículo se exponen aspectos relacionados con las ideas de las profesoras ${ }^{4}$ participantes en este estudio acerca de lo que, desde sus perspectivas teóricas y prácticas, son los métodos de enseñanza de la lectura y la escritura, la bondad de su aplicación, las condiciones de su puesta en práctica, como la preparación de clases, la evaluación del aprendizaje y las consecuencias que generan, expresadas por ellas como dificultades. Está organizado en cuatro partes. En la primera se exponen brevemente algunos antecedentes de investigaciones sobre los métodos de enseñanza de la lectura y la escritura, $y$ lo que piensan los profesores sobre sus usos. En la segunda se explica cuál fue la metodología utilizada en la investigación. En la tercera parte se presentan los resultados y su discusión, y en la cuarta se exponen los hallazgos.

\section{Antecedentes}

Para empezar, es necesario señalar que a pesar de que la investigación sobre la alfabetización inicial y los procesos de lectura y escritura en los últimos años se ha centrado en aspectos diferentes al uso de los métodos de enseñanza, en la práctica los

3 En este documento, cuando se habla de niños se hace referencia a los niños y las niñas.

4 Se habla de profesoras porque todas las participantes en este proceso de investigación fueron mujeres. profesores siguen mostrando apego a ellos. Fue esta la situación que motivó la decisión de tomar como una de las categorías de análisis en la investigación mencionada los métodos de enseñanza de la lectura y la escritura.

Con respecto a las investigaciones sobre el uso de diferentes métodos de enseñanza, en América Latina son bastante conocidos los trabajos pioneros en las décadas de los setenta y ochenta, adelantados por Berta Braslavsky, María Carbonell de Grompone y Mabel Condemarín, entre otros. A partir de ellos, ya se empezaba a vislumbrar que el aprendizaje de la lectura y la escritura es algo que va mucho más allá de la aplicación de un método de enseñanza. Esta idea se corroboró de manera contundente con las investigaciones hechas por Ferreiro y Teberosky (1979), en las que se comprobó que la propuesta de enseñanza basada en los métodos tradicionales desconocía tanto la actividad de construcción cognitiva que se da en el niño a lo largo de ese proceso, como los usos sociales de la lectura y la escritura.

Sin embargo, en años posteriores a las investigaciones de Ferreiro y Teberosky (1979), todavía se encuentra en la literatura sobre este tema alusiones al uso y a los efectos de la enseñanza de la lectura y la escritura mediante la aplicación de diferentes métodos (Unesco, 1986; Caldera y Escalante, 2009; Córdova y Ochoa, 2008, entre otros), lo que muestra cierta resistencia en la práctica docente a asumir los procesos de construcción de la lengua escrita como resultado de la actividad cognitiva del niño. Investigaciones recientes como la de Jiménez y O'Shanahan (2008) han encontrado que los profesores se basan en tres enfoques para enseñar a leer y a escribir a sus estudiantes: uno, basado en la metodología de Freinet, otro a partir de la enseñanza de los fonemas y el último a partir de la enseñanza de sílabas.

Por su parte, Tolchinsky (2008) identificó, en una amplia muestra tomada en España, tres perfiles de profesores con respecto a sus prácticas docentes, según las características de los procesos de alfabetización que adelantaban en la escuela: instruccional, multidimensional y situacional. La mayor parte de los profesores se caracterizó como instruccional, lo que implica reconocer que trabajaban preferencialmente 
con los métodos fónico, silábico, global o mixto. En esta misma línea, Hernández (2012) encontró en su investigación que la enseñanza de la escritura en el primer grado se apoya en ejercicios de planas, la copia y el dictado con buena letra.

Podrían mencionarse más investigaciones sobre esta temática, con resultados similares, que por razones de espacio no se referencian aquí. Parece ser que a pesar de que los profesores han incorporado a sus discursos elementos novedosos que hacen referencia a la importancia de tomar en cuenta al niño, los métodos de enseñanza aparentemente se anclaron en las prácticas cotidianas, convirtiéndose en el eje de trabajo en el aula como respuesta a las necesidades de enseñanza, aunque no necesariamente a las de aprendizaje. De aquí la importancia de investigar sobre cómo y qué aprenden los profesores sobre los métodos de enseñanza de la lectura y la escritura.

\section{Metodología}

Como ya se mencionó, la pregunta de investigación fue ¿cómo aprenden los profesores a diseñar estrategias que faciliten la alfabetización inicial ${ }^{5}$ ? Una de las categorías de análisis en este proceso fue el aprendizaje sobre los métodos de enseñanza de la lectura y la escritura. El objetivo principal fue buscar comprensiones sobre las maneras en que los profesores relacionan su saber teórico con el práctico para dar respuesta a sus necesidades en el aula. Por la naturaleza de la pregunta de la investigación, se trabajó con una metodología cualitativa, dado que, según Bonilla y Rodríguez (2000, p. 63),

La vida cotidiana se mantiene porque se concreta en rutinas institucionalizadas y se reafirma continuamente en la interacción del individuo con otros individuos. La realidad se internaliza y permanece en la conciencia, mediante procesos sociales que son posibles gracias al manejo de diferentes niveles de conocimiento que informan sobre las acciones que emprenden los individuos. Estos niveles de conocimiento, implícitos en el

5 En este contexto, facilitar la alfabetización inicial implica desarrollar en los niños la oralidad y favorecer su acercamiento a los usos sociales de la lectura y la escritura. comportamiento de los miembros de una sociedad, configuran la dimensión cualitativa de esta realidad.

El alcance de esta investigación fue descriptivo y exploratorio. Se trabajó durante ocho meses en un proceso de formación de las profesoras en la facilitación de la alfabetización inicial, con una muestra intencional y estratificada constituida por 25 educadoras del nivel inicial (jardín, transición y primer grado) tanto del sector oficial (urbano y rural) como del privado, madres comunitarias ${ }^{6}$ y estudiantes de últimos semestres de Pedagogía Infantil de la Universidad de La Sabana. Con esta conformación de la muestra se buscaba que estuvieran representados diferentes grados escolares, el sector oficial y el privado, las zonas rural y urbana, y educadoras sin formación académica, profesoras en proceso de formación inicial (que estuvieran cursando los últimos semestres del pregrado) y profesoras en ejercicio.

Como instrumentos de recolección de información se utilizaron el cuestionario, la entrevista semiestructurada, el grupo focal, las videograbaciones de clases y los talleres de análisis de las videograbaciones. En la construcción de las categorías emergentes se acudió al análisis de contenido.

El procedimiento investigativo se llevó a cabo de la siguiente manera: se partió de identificar por medio de cuestionarios y entrevistas semiestructuradas los conocimientos y las concepciones que traían las educadoras con respecto a lo que implica facilitar la alfabetización inicial en los niños. Con esta información se construyeron categorías emergentes que se constituyeron en insumos para generar una matriz de análisis que permitiera la comprensión de nuevas informaciones que se fueran recogiendo en el proceso. Para cada profesora se tomaron tres situaciones de clase elegidas libremente por cada una, que fueron filmadas y que después fueron motivo de análisis en talleres desarrollados en grupo. Se analizaron 75 situaciones de clase; la información

6 En Colombia las madres comunitarias son mujeres que se encargan de cuidar los hijos de otras madres que van a trabajar. Inicialmente se encargaban solamente de cuidar a los niños, pero gradualmente se han ido incorporando a la formalidad de la atención a la primera infancia, con lo que han asumido otras funciones educativas. 
derivada de este análisis se registró en la matriz elaborada al inicio del proceso. Posteriormente se hicieron grupos focales para discutir los hallazgos.

Para finalizar los análisis se hizo la reconstrucción teórica, jerarquizada en temas, categorías, relaciones entre categorías y comprensiones. En este análisis se presentaron resultados tanto comunes a todas las educadoras, como específicos para cada tipo de población, según el nivel en que enseñaban (jardín, transición o primero), el sector en que trabajaban (público o privado), el área en que se desempeñaban (urbano o rural), el tipo de formación (madres comunitarias, estudiantes de Pedagogía Infantil, profesoras con pregrado o con posgrado), y los años de experiencia (entre 2 y 24 años).

\section{Resultados y discusión}

\section{El conocimiento de los métodos}

Para iniciar este apartado, es importante retomar los aportes de Litwin (2008), ya que esta investigadora precisa que cuando se habla de métodos de enseñanza no necesariamente se aborda este tema como tal, sino que se alude a las técnicas empleadas para enseñar, en lo que se reconoce como instrumentalismo y que conduce a seguir unos pasos de manera prescriptiva y mecánica, sin atender a las características de los estudiantes ni a las particularidades de cada contexto. En la misma línea, Camilloni et al. (2008, p. 79) afirma que "De ahí proviene una visión simplificada de lo metodológico en didáctica, como un modelo de instrucción basado en técnicas que predefinen pasos organizados rigurosa y linealmente para lograr diseños que permitan obtener productos óptimos y válidos en diferentes contextos". Sin embargo, el método es otra cosa, pues, como señala Litwin (2008, p. 68), "Referirse al método implica recuperar su definición clásica, como la manera ordenada y sistemática de enseñar. Referirse al método, desde su origen, es referirse a un constructo de la filosofía".

Para el caso que nos ocupa en los resultados de esta investigación, es necesario señalar que las referencias que se encontraron al uso de los métodos se enmarcan dentro de una posición instrumentalista, como puede corroborarse a lo largo de las afirmaciones hechas por las profesoras con quienes se trabajó, tanto desde el ámbito teórico, como desde las reflexiones suscitadas a partir de sus prácticas. Así, afirman: "El fonético enseña sonidos, permitiendo crear una representación mental" (P2 PM); "Prejardín, para leer se muestra un cuento con dibujos grandes y el niño puede como leer el cuento. Para escribir, que ellos observen las letras y las transcriban" (P1 CM); "Poniendo palabras completas que entiendan qué dice y vean cómo se escribe" (P5 CM).

El conocimiento sobre los métodos de enseñanza de la lectura y la escritura de las educadoras en formación es prácticamente inexistente, con una manifestación de desprecio hacia ellos, a través de sus discursos, lo que lleva a hacer afirmaciones como: "No hay método como tal. Todos los niños aprenden de forma diferente. Lo mejor es utilizar varios métodos" (E3 PI); "No tengo método" (E4 PI); "El global, combinado, inductivo, deductivo. Me gusta trabajar con el silábico ya que sabiendo los sonidos más fácil forman las palabras, escriben y leen" (P5 DC).

Las referencias con respecto a la enseñanza se hacen desde las actividades que planean para que los niños estén contentos y así se asegure su aprendizaje. Lo anterior implica reconocer la fuente y el sustento para la organización de los aprendizajes de los profesores acerca de las formas de lograr, a su vez, aprendizajes en los estudiantes en otro lugar, que en este caso se identificó con el buen estado emocional de los niños, como se evidencia en las siguientes intervenciones: "Que sea emocionante y llamativa para que tenga un objetivo muy concreto" (P2 MP); "La motivación, como buscar que ellos mismos creen la clase, la formen, estén felices y aprendan jugando" (P3 CR). Esta idea estaría sustentando en las educadoras la importancia de acercarse a ellos de manera lúdica y amorosa, para garantizar el éxito en el aprendizaje.

Puede decirse entonces que para las profesoras en formación el tema de los métodos de enseñanza es bastante oscuro y difuso: "No he tenido la oportunidad de usarlos y no recuerdo el nombre" (E1 PI). Como no los conocen en detalle, no están 
en condiciones de analizarlos y mucho menos de evaluarlos para tomar una posición frente a ellos. Si bien en este momento se sabe que la enseñanza de la lectura y la escritura no es una cuestión de métodos (Ferreiro y Terebosky, 1979) —entendidos en una perspectiva instrumentalista-, sí es importante que los futuros educadores los conozcan para comprender las propuestas alternativas de enseñanza que han surgido a lo largo de la historia para enseñar a leer y a escribir. Así mismo, este conocimiento puede constituirse en una base para tomar decisiones al llegar a las instituciones que tienen definidos sus enfoques y métodos de enseñanza, imponiéndolos a los profesores que trabajan en ella. Vale la pena anotar que, a pesar del desconocimiento expresado acerca de los métodos de enseñanza, las profesoras en formación conservan algunas huellas de las enseñanzas recibidas en la universidad, ya que afirman que los métodos deben adecuarse a las necesidades de los niños: "No existe mejor o peor método, este se debe ajustar a las necesidades y contexto del niño" (E2 PI).

En los discursos de algunas de las estudiantes de Pedagogía hay alusiones a que no utilizan los métodos porque los niños son muy pequeños. Esta idea resulta interesante, ya que sugiere que desde la perspectiva de las profesoras en formación el uso de los métodos de enseñanza de la lectura y la escritura corresponde a una etapa formal, que, a su vez, se correspondería con una edad determinada y con un grado escolar definido. Esto tiene consecuencias en la progresión del proceso de aprendizaje de la lectura y la escritura, pues llevaría a pensar que en el preescolar o hasta un grado particular su desarrollo es libre, espontáneo y sin intervención estructurada de parte del profesor, y de ahí en adelante se hace de manera más formal, con lo que se sugiere la comprensión del método de enseñanza como algo prescriptivo, que obliga a encuadrar la enseñanza y el aprendizaje en procesos rígidos: "Silábico, fonético, global, alfabético. No se usarlos bien, pero uno mezcla un poquito de cada uno" (P2 PM).

Esta situación difiere de la de las profesoras en ejercicio, quienes sí hacen alusión a los métodos de enseñanza de manera precisa y en la mayoría de los casos están en condiciones de explicar en qué consisten. En otros casos se evidencia confusión entre los métodos y se encuentra mezcla en sus explicaciones de elementos de uno y de otro, sin evidenciar claridad al respecto: "Fonológico, global, lectura de imágenes y gestual, el niño utiliza su imaginación y crea un cuento" (P4 PM). Se hace alusión a la bondad de mezclar métodos para obtener mejores resultados en la enseñanza de la lectura y la escritura. Sin embargo, se evidencia confusión en las denominaciones y en sus propuestas. Se refieren a ellos con nombres específicos, enuncian algunos detalles aislados de algunos métodos que recuerdan haber oído mencionar, pero no hay ninguna alusión a sus postulados epistemológicos ni a sus lógicas internas, ni se refieren de manera explícita a lo que implica el manejo de cada uno de ellos; es decir, no hay referencias precisas a los métodos de enseñanza de la lectura y la escritura, ni desde la perspectiva epistemológica, ni desde la instrumental: "El combinado y también el globalizado, según los intereses y necesidades" (P3 DC); "El mejor método es... todos son un conjunto de herramientas muy valiosas, desde el global hasta silábico y fonético pero el mejor es escritura espontánea, dejar que ellos mismos reproduzcan" (P3 CR). Por lo tanto, hay que relativizar esta idea de que conocen los métodos, pues no es lo mismo saber nombrarlos que conocer su estructura, su lógica y sus propósitos. Por otra parte, sus clasificaciones no son nítidas, en tanto se refieren a los métodos tradicionales de enseñanza de la lectura y la escritura por oposición al silábico, el cual también se reconoce como tradicional; es decir, la categoría de tradicional no informa nada preciso en este caso.

Al preguntar por el mejor método de enseñanza de la lectura y la escritura, estas profesoras expresan el reconocimiento de la importancia de que el método utilizado promueva la participación activa de los niños en el proceso, pero afirman que el mejor es el que muestra muchas imágenes, láminas y palabras de uso frecuente, las cuales enfatizan la importancia de la percepción visual y de la repetición en el aprendizaje de la lectura y la escritura. Si bien, ninguna de las profesoras mencionó que el aprendizaje de la lectura y la escritura se llevara a cabo fundamentalmente a partir de la observación 
y copia de las letras, sí es evidente en este énfasis en la percepción visual y en la recordación de palabras la idea subyacente de que se aprende mirando las letras, de ahí la importancia que le conceden a este material: "El tradicional, donde les muestro el dibujo, lo observan, escriben la palabra, la observan y la leen y luego les doy el sonido del fonema de las vocales" (P5 DC); "Mirar cómo explicarles a los niños. Ejemplo: mostrar el nombre de la fruta con letra clara y grande para que relacionen el objeto con lo escrito" (P1 DC).

Por otra parte, las profesoras señalan que todos los métodos aportan algo bueno. El mejor, según afirman, es el que permite la escritura espontánea para que los estudiantes reproduzcan: "Buscar cuál de todos los métodos le gusta al niño, con cuál se identifica y trabajar el más acorde a sus necesidades y contexto" (P3 CR). Esta afirmación sugiere nuevamente la idea de que se aprende copiando, contrariamente a lo que se pensaría al tomar en cuenta el concepto de escritura espontánea, que fue claramente señalado por Ferreiro y Teberosky (1979), como una expresión de aquello que los niños están en capacidad de construir con respecto al sistema de escritura, y a partir de la cual generan y prueban hipótesis propias que no han sido enseñadas por los adultos. Una vez más, llama la atención el uso de ciertas expresiones que remiten a conceptos diferentes del que fue elaborado originalmente.

A pesar de afirmar que el método de enseñanza de la lectura y la escritura lo define la institución en que trabajan y, por lo tanto, deben acogerse a este, hay alusiones al uso de diversos métodos para adecuarlos a las necesidades de los niños. También hay referencia a los métodos tradicionales, pero de una manera confusa, pues les atribuyen características que no les corresponden.

Por otro lado, tanto en las profesoras en formación como en quienes están en ejercicio es notorio su desconocimiento de los elementos lingüísticos y cognitivos implicados en el aprendizaje de la lectura y la escritura, pues ubican toda la garantía de éxito para este proceso en el estado emocional y la motivación de los niños, con lo cual dan a entender que en este proceso se alcanza el éxito solamente con buena voluntad de generar un ambiente agradable: "Para que los niños aprendan hay que ser amable, brindar buen ambiente, tener confianza, dominio en sí mismo, ser alegre, paciente y amoroso" (P4 PM).

En el grupo de las madres comunitarias resaltan algunos aspectos interesantes y diferentes de los otros grupos de educadoras. En primer lugar, sus comentarios sobre los métodos de enseñanza están referidos explícitamente a las actividades que desarrollan cotidianamente con los niños. Si se compara con lo dicho por los otros grupos, se puede constatar que en todos ellos se hace este tipo de referencias. La diferencia radica en que en los otros grupos se busca la manera de hacer coincidir estas actividades con lo que consideran que proponen teóricamente los diferentes métodos, con lo cual esperan acomodar sus discursos a la teoría conocida, mientras que las madres comunitarias no lo hacen.

Esta posición ya se había destacado en otro trabajo anterior en el que afirmamos que tanto las profesoras como las madres comunitarias "ignoran la pedagogía, adjetivan la didáctica y sustantivan la actividad" (Guzmán y Ecima, 2011, p. 10). Planteamos entonces que es posible que, a través del análisis de las prácticas en el aula y de la planeación de actividades, se logre precisar lo que los profesores saben acerca de los métodos, pues, según plantea Litwin (2008, p. 67), "planear la actividad implica generar un proceso reflexivo y de construcción del conocimiento que incluye el mismo desafío que el que se le planteará al alumno". Vale la pena aclarar que en este proceso de planeación "mezclando métodos" se da lo que Gloria Edelstein (citada en Litwin, 2008) llama "construcción metodológica" (p. 68), en tanto las profesoras, a partir de lo que han aprendido en la teoría, pero también de lo que les dice la práctica, hacen adecuaciones que consideran pertinentes para enseñar. La pregunta acerca de qué es lo que hace que lo consideren pertinente nos remite a sus ideas acerca de lo que es enseñar a leer y a escribir.

Sus ideas acerca de cómo aprenden los niños a leer y a escribir están muy relacionadas con la copia y con la observación, lo que no se aparta mucho de lo dicho por los otros grupos. Sin embargo, se hace referencia a posiciones actuales con respecto 
al acercamiento inicial que se propone que los niños hagan a la lectura y la escritura; afirma una profesora: "Que el niño tenga el contacto directo con los textos, símbolos y logos”. Una vez más, se resalta el hecho de que conocer algunas expresiones y usar ciertos términos no garantiza que el concepto que le subyace esté presente; el conocimiento de lo que saben los profesores implica conocer sus discursos, pero especialmente la relación entre ellos y las prácticas a que dan lugar.

\section{La planeación de las clases}

Las educadoras en formación afirman que lo primero que hacen al planear una clase es tener en cuenta los objetivos de la sesión y la motivación que deben despertar en los niños, es decir que no se evidencia una perspectiva del proceso, sino que la atención y la energía se centran en la forma de la clase. Ninguna se refirió al contenido de esta. Consideran que a través de la planeación de las clases pueden programar actividades que les permitan identificar las dificultades de aprendizaje de sus estudiantes; asimismo pueden programar que los niños se diviertan mientras aprenden. Con respecto a la primera idea, parece existir en las profesoras en formación, dentro de sus concepciones de aprendizaje de la lectura y la escritura, la idea de que allí es bastante probable que se presenten problemas.

Por otra parte, en las profesoras en ejercicio se perciben confusiones sobre lo que plantean los diferentes métodos de enseñanza de la lectura y la escritura con respecto a lo que generan en los niños. Como producto de esta situación, ante los escasos o deficientes resultados de la aplicación de métodos, deciden inventar uno propio. Esta posición resulta muy interesante, en tanto da cuenta de la recursividad de las profesoras para resolver dificultades que se les presentan en su vida cotidiana, acudiendo a su creatividad para generar construcciones metodológicas, como ya se planteó en este documento. Otro aporte interesante de este grupo de profesoras es la afirmación de que cuando los niños regresan de las terapias a las que han sido remitidos por confundir las letras o por trabajar despacio se necesita volver a motivarlos para la lectura y la escritura. Lo anterior sugiere la discontinuidad entre los procesos de enseñanza de la lectura y la escritura y las terapias que buscan remediar las dificultades en ellos, lo que ameritaría una reflexión sobre la pertinencia de enviar a los niños a estas sesiones de trabajo o de articular la práctica pedagógica con la terapéutica, pero esta no se presentó.

Para preparar la clase tienen en cuenta que sea motivadora, que tenga un objetivo concreto, que despierte el interés en los niños, que responda a sus necesidades, que vehiculice la intención del profesor y que se cuente con un buen material que estimule los sentidos. Nuevamente encontramos decisiones metodológicas basadas en la idea de que la lectura y la escritura requieren fundamentalmente el trabajo perceptual. Las profesoras en ejercicio que trabajan en el sector privado, con respecto a la preparación de la clase, destacan la importancia de tener en cuenta en su estructura lo lúdico, la participación de los niños y su producción. Resaltan además las bondades del trabajo cooperativo. Consideran que lo más importante en la preparación de las clases es el material, que consiste en letras grandes "para que los niños las puedan ver bien" (P3 CR). Lo anterior sugiere una vez más que el éxito de la enseñanza para la alfabetización inicial encuentra su principal soporte en el uso de materiales que estimulen sus sentidos. Sin embargo, también hay alusiones tanto a las necesidades, a las habilidades y a los intereses de los niños, como a las estrategias, pero sin definirlas. Dentro de estas actividades incluyen la evaluación, aspecto que se presenta en seguida.

\section{La evaluación del proceso de aprendizaje en los niños}

Con respecto a la evaluación del aprendizaje de la lectura y la escritura es evidente que las profesoras no tienen criterios claros para hacerla; de hecho la consideran una actividad más, como se señaló en el párrafo anterior. La relacionan con la tranquilidad y el disfrute, de tal manera que consideran que el proceso va bien si los niños están contentos; también la relacionan con la comprensión clara, pero no especifican qué es. Señalan que se dan cuenta que 
los niños están aprendiendo bien cuando la lectura y la escritura les resultan significativas y las disfrutan. Sobre la evidencia de que sus estudiantes están aprendiendo bien, aluden a que esto se da cuando los papás informan que el niño está leyendo de manera libre y espontánea, y además lo disfruta. Si bien la evaluación es algo que debería darse en el ámbito escolar, esta afirmación remite al reconocimiento implícito de la funcionalidad social de la lengua escrita, pero sin claridad con respecto a qué es lo que hay que evaluar en los niños, ni cómo hacerlo, ni mucho menos qué hacer con los resultados de la evaluación. Se enfatizan nuevamente aspectos emocionales y de actitud para dar cuenta del aprendizaje, es decir que evidencian la consecuencia del aprendizaje, pero no el proceso, ni retoman sus resultados durante la enseñanza. Ninguna mencionó que la tomara como objeto de análisis y reflexión para preparar sus clases siguientes.

Tampoco las madres comunitarias tienen claridad sobre lo que se debe evaluar en los niños para dar cuenta de sus progresos. Al igual que mencionaron las profesoras de los otros grupos, la evidencia se da cuando ya los niños pueden leer y escribir solos. Esta falta de claridad sobre la evaluación en los niños y en las estrategias de enseñanza conduce a que determinados comportamientos de los niños se interpreten como dificultades de aprendizaje, como se presenta a continuación.

\section{Las dificultades en el aprendizaje}

Particularmente las madres comunitarias con respecto a las dificultades en el aprendizaje de la lectura y la escritura consideran que a los niños no hay que forzarlos en este proceso y que hay que dejarlos que escriban como puedan, lo que guarda una interesante proximidad con las escrituras espontáneas propuestas desde 1979 por Ferreiro y Teberosky. En esta misma línea, señalan que para superarlas hay que "Presentarles alternativas más sencillas y hacer un buen acompañamiento" (MC3 CM), lo cual es muy interesante, ya que remite a tener en cuenta al niño como centro del aprendizaje. Al igual que las profesoras de los otros grupos, piensan que a partir de la percepción visual los niños pueden aprender más fácil; esa idea sustenta afirmaciones referidas al tamaño de la letra para facilitar el aprendizaje de la lectura y la escritura. Hay alusión reiterada a las dificultades que tienen los niños para aprender a leer y a escribir que se basan en sus capacidades individuales relacionadas con la percepción, la asociación y la discriminación, lo que lleva a que confundan las letras; sin embargo, afirman que es necesario "revisar en qué proceso cognitivo están fallando: asociación, atención [...]” (P1 DC). Llaman la atención dos aspectos en esta afirmación. Por una parte, la alusión a los procesos cognoscitivos, que no había aparecido antes en los grupos de profesoras. Por otra parte, la alusión a "revisarlos", que parece indicar que consideran que ellos tienen incidencia en los procesos de aprendizaje de la lectura y la escritura como prerrequisito y no como consecuencia del proceso de desarrollo y de aprendizaje. Esto sugiere la idea de que la enseñanza debe seguir al desarrollo biológico. También señalan como dificultad en este proceso la lentitud que se da en algunos niños, aunque, curiosamente, de manera paralela hablan de los diferentes ritmos de aprendizaje.

Al hablar de las dificultades, las madres comunitarias se refieren a tropiezos en el proceso de enseñanza, mas no a dificultades en el aprendizaje debidas al desarrollo de los niños. Afirman que en estos casos de dificultad es el profesor quien debería apoyar a los niños al buscar nuevos recursos de enseñanza y acudir a otros métodos que pueden resultarles más efectivos. Aseveran que en caso de que se presenten dificultades en el aprendizaje de la lectura y la escritura en los niños, el profesor debe estar en capacidad de identificarlas para buscar ayuda interdisciplinar. Plantean que es posible evitarlas si se responde a los intereses de los niños. En esta afirmación se diferencian de las que hacen las profesoras, para quienes, como ya se mencionó antes, las dificultades obedecen a características particulares de los niños.

\section{Hallazgos}

A partir de la información presentada, pueden derivarse las siguientes conclusiones: 
Sobre el conocimiento de los métodos de enseñanza de la lectura y la escritura

Las profesoras conocen los métodos de enseñanza de la lectura y la escritura a través de las prácticas. Si bien las educadoras participantes en esta investigación, con excepción de las madres comunitarias, han tenido formación académica en la que se les han enseñado los métodos de enseñanza de la lectura y la escritura, parece ser que este conocimiento emerge a partir de la experiencia práctica, ya que son las educadoras en ejercicio quienes afirman conocerlos y aplicarlos. Lo anterior sugiere la necesidad de construir saberes didácticos fundamentados en las prácticas, pues es desde allí que se hace posible poner en uso los conocimientos teóricos, para confirmarlos, rechazarlos o modificarlos según los resultados que arroje su aplicación. Buena parte de este saber parece venir incluso del sentido común, entendido como

[...] una forma de conocimiento colectivo de carácter práctico: a través de la construcción de representaciones, en las luchas de clasificación de la realidad, en las que se pretende poseer el monopolio del orden y la nominación, se establecen categorías que, cuando son aceptadas ampliamente, por la vía de un proceso de naturalización del orden dominante y de sus relaciones, ganan validez general. (Márquez, 2008, p. 13).

La validez general de este saber estaría dada por el éxito alcanzado en las aplicaciones que hacen de diversos métodos e incluso por la mezcla de ellos. Seguramente en su generalización incidan los resultados en el aprendizaje de los estudiantes, las prácticas propias y las de otros, los comentarios de los padres, las nuevas informaciones, las conversaciones formales e informales que sostienen las educadoras en sus ámbitos de trabajo cotidiano, pues, tal como lo señala Mercado (2002), los saberes pedagógicos se construyen a partir de diferentes voces.

Una de estas voces, que aún no ha sido completamente explorada, es la práctica. Al respecto afirma Verónica Edwards (citada en Rockwell, 1995):

Más específicamente lo que se pone en juego en la interacción de maestros y alumnos es una determinada lógica de la enseñanza y de la participación formal de los alumnos. Esta constituye el contenido implícito que se va transmitiendo a lo largo de la lección y que les da un sentido particular a los diversos contenidos específicos que la lección incluye. A través de su participación, los alumnos objetivan su comprensión de lo que es "conocer" o "aprender" y aportan contenidos específicos a la clase. Estas definiciones forman parte de la dinámica en la cual se concretan la presentación y la apropiación del conocimiento. (p. 149).

En la interacción del maestro con sus alumnos, él se da cuenta que los métodos de enseñanza que aprendió en la universidad, puestos en práctica tal como se los enseñaron, no funcionan siempre ni con todos los niños. Es a través de dicha interacción que el profesor valida la mezcla de sus métodos. En esa misma perspectiva encontramos la declaración de Edelstein (citada en Litwin, 2008), quien afirma que la categoría de construcción metodológica, en el sentido en que se señaló antes en este artículo, daría cuenta de las adecuaciones que hacen los profesores para atender las particularidades del contexto en el que trabajan y que sería allí en donde tendríamos que indagar acerca de lo que hacen los profesores con los métodos que conocen. Esta propuesta de reconocer los saberes que se derivan de las prácticas cotidianas y de la interacción del maestro con sus alumnos ya ha sido planteada por Rockwell (1995), quien afirma que generalmente el conocimiento derivado de la práctica se descalifica frente al producido por los académicos que trabajan en las universidades, con lo cual pierde el potencial de retomarlo como fuente de aprendizaje práctico de los profesores.

Las ideas arriba expuestas refuerzan la necesidad de seguir explorando las prácticas cotidianas de los maestros como fuente de sus saberes, y tratar de comprender sus lógicas y sus racionalidades, que no son las mismas del saber académico estructurado y organizado. Quizá esto encuentre alguna explicación en lo dinámico de las prácticas pedagógicas y tenga un referente en las educadoras cuando afirman que usan un método determinado o incluso que no los conocen, pero que hay que adecuarlos a las necesidades de los niños. Esto nos llevó a analizar 
nuestros resultados desde lo que significan las prácticas pedagógicas para estas educadoras y no desde el saber académico, que las interpretaría como caóticas, amorfas y sin sustento, con lo cual ignoran el análisis del significado que adquieren para las profesoras en su cotidianidad.

En esta misma línea, Litwin (2008) afirma que la complejidad de los diversos contextos en que trabajan los profesores favorece la toma de conciencia de lo que hacen, para determinar cómo sus intervenciones implican rupturas o validación de sus conocimientos. Es decir, los profesores en esa interacción cotidiana en la que llevan a cabo sus prácticas estarían haciendo lo que Litwin llama la reconstrucción metodológica, proceso que amerita ser estudiado y analizado a profundidad dada la complejidad que revisten los procesos de enseñanza y de aprendizaje.

\section{Sobre la concordancia entre el uso de métodos y los procesos de aprendizaje}

Respecto a la concordancia que se esperaría encontrar en una situación ideal entre los métodos de enseñanza y los procesos de aprendizaje de los niños, desde una didáctica pretendidamente universal, se evidencia a través de los discursos de las educadoras, sustentados en su experiencia práctica, que esta no ocurre. Por esa razón mezclan los métodos en un intento por hacer coincidir sus propuestas de enseñanza con las necesidades de aprendizaje de sus estudiantes. Una situación que parece dificultar la referencia clara a una construcción metodológica es el desconocimiento que tienen las educadoras con respecto a lo que implica para los niños aprender a leer y a escribir, así como de la naturaleza misma de la escritura como sistema de representación. Para ellas, en la aplicación práctica, todavía la lectura y la escritura se aprenden por medio de la visión, de ahí que le asignen tanta importancia a los carteles y al tamaño de las letras, y que se refieran más a las actividades que a una propuesta didáctica articulada.

Por otro lado, esta situación sugiere la inadecuación de los métodos - en su versión instrumentalista- para apoyar los procesos de enseñanza y de aprendizaje de la lectura y la escritura. Por ello no encuentran esta coherencia entre la enseñanza y el aprendizaje, pues si bien el segundo no es el reflejo de la primera, sí debería encontrarse alguna relación entre los dos procesos. Sin embargo, resulta curioso que, a pesar de no encontrarse coherencia, se sigan utilizando los métodos referidos para enseñar a leer y a escribir. De otro lado, parecería que tampoco es posible hablar en este caso de reconstrucciones metodológicas en tanto no se evidencia una metodología, sino una serie de actividades en las cuales el énfasis está puesto en los recursos utilizados para las clases y en el afecto que la profesora dé a los estudiantes. Esta falta de claridad probablemente dificulta mucho el dar cuenta de las razones de los avances de los estudiantes mediante los procesos de evaluación, aspecto que se expone en seguida.

\section{Sobre la evaluación}

Este resultó ser uno de los aspectos más reveladores de este proceso de investigación, dado que las referencias que se hicieron a la evaluación tenían que ver con el resultado y no con los procesos. Estos resultados pueden ser reportados tanto por las educadoras como por los padres de familia, quienes les informan que sus hijos ya leen solos. Este aspecto es muy importante porque parece que las educadoras no tienen manera de hacer seguimiento a los procesos de los niños y mucho menos tener control sobre él por medio de sus propuestas didácticas. Pareciera que la enseñanza siguiera un camino paralelo al del aprendizaje y, por consiguiente, nunca se tocaran. Por otra parte, las evidencias que las educadoras manifiestan encontrar están más relacionadas con estados emocionales y afectivos de los niños que con las posibilidades que tengan de construir sentido a partir de lo que leen y de la posibilidad que tengan de comunicarse por medio de la escritura. Es decir, los avances en el aprendizaje de la lectura y la escritura quedan a la deriva, pero las educadoras intentan favorecerlos mediante las expresiones de cariño y las voces de aliento a sus estudiantes; esta situación por supuesto es deseable, pero no da cuenta por sí misma de la orientación de los procesos de aprendizaje. 
Es interesante observar que la imprecisión de las educadoras participantes en esta investigación, con respecto a los métodos de enseñanza de la lectura y la escritura, es consecuente con el desconocimiento sobre la naturaleza del objeto de estudio en la alfabetización, ya que, como se mencionó en este artículo, relacionan el éxito en la enseñanza con el uso de materiales que "tengan las letras grandes". En consecuencia, desde su perspectiva, la evaluación debe orientarse a constatar que los niños reconocen y reproducen las letras, lo que se ha demostrado mediante la investigación que no es garantía de estar alfabetizado (Ferreiro y Teberosky, 1979).

En esa línea se sitúan los aportes de Katzkowicz (citada en Anijovich, 2010, p. 114), al afirmar que las creencias de los profesores inciden en sus formas de evaluar. Para el caso de esta investigación resulta tan evidente la falta de articulación entre lo que proponen en el aula de clase y los resultados que el referente de aprendizaje de la lectura y la escritura de los estudiantes es la información de los padres, quienes dicen que "ya puede leer solo". No obstante, mientras que esto sucede, lo que pasa en el proceso se interpreta como un problema, que debe ser asumido por los estudiantes e incluso por sus familias, como se explica a continuación.

\section{Sobre los problemas}

Según los análisis sobre lo que las educadoras reportan respecto a los procesos de aprendizaje de la lectura y la escritura, consideran parte del proceso mismo la presencia de problemas. Dichos problemas son atribuidos a los niños y a las situaciones de sus familias. Explican los problemas por la desnutrición, la disfuncionalidad de la familia, la desmotivación de los niños, la falta de interés y los problemas físicos, entre otros, pero no hubo ninguna referencia a que esto les atañera a los profesores, que son quienes definen cómo enseñar a leer y a escribir, lo que deja en clara desventaja a los niños provenientes de contextos en los que poco o nada se usan la lectura y la escritura.

Lo anterior permitiría afirmar que las educadoras ven los problemas en el proceso de aprendizaje de la lectura y la escritura con carácter de exterioridad a la didáctica; sin embargo, se encuentra una fuerte contradicción cuando afirman que parte de los problemas se deben a que los niños vienen mal preparados del curso anterior. Entonces, la pedagogía y la didáctica sí inciden en el proceso de aprendizaje cuando tiene que ver con otros profesores, pero no cuando se trata de cada una de ellas.

En este orden de ideas, se asumiría además que a cada grado escolar le corresponde un particular nivel de desarrollo con respecto al aprendizaje de la lectura y la escritura, lo cual lleva a esperar un resultado homogéneo en los estudiantes. Esta creencia vendría acompañada de otra, que llevaría a sustentar que, al no darse los desarrollos esperados en los grados anteriores, resultaría inevitable reconocer que quienes no los alcanzaron estarían condenados a la presencia de problemas en el aprendizaje y por consiguiente al fracaso escolar.

Los resultados de esta investigación plantean interesantes retos para la formación de los profesores, en tanto muestran la necesidad de analizar cuidadosamente la forma en que ha venido formándose a los educadores para enseñar a leer y a escribir a los niños, ya que, a pesar de los esfuerzos que vienen adelantándose en diversos lugares y de los resultados arrojados por la investigación básica, todavía falta mucho camino por recorrer, especialmente en el estudio y análisis de los aprendizajes de los profesores que se derivan de las prácticas cotidianas en el aula. Este aspecto, que convoca a todas las instituciones formadoras de docentes, requiere de dos abordajes: uno orientado hacia aquellas prácticas exitosas que parecen superar lo planteado en la teoría, y el otro dirigido hacia las prácticas que los profesores intentan explicarse desde teorías conocidas, a pesar de permanecer ancladas en la tradición.

Así mismo, vale la pena estudiar cómo se articula el aprendizaje académico de los profesores durante su formación en la universidad, con sus creencias iniciales y con las construcciones metodológicas que hacen a lo largo de sus prácticas. Con respecto al conocimiento y manejo de los métodos de enseñanza de la lectura y la escritura, también conviene revisar tanto sus fuentes epistemológicas, como sus contextos de aplicación, para situarlos de una 
manera provechosa en las rutas de formación de los futuros profesores.

Por otra parte, en los niveles de formación avanzada, como las especializaciones y las maestrías, se requiere proponer trabajos de grado que recuperen las prácticas cotidianas en el aula para reflexionar sobre ellas y buscar el sentido que tienen dentro de contextos particulares y articularlas con la teoría existente para buscar las tensiones, las rupturas, las inconsistencias y las continuidades. De esta manera se lograría rescatar la práctica como fuente de conocimiento pedagógico. No hay que olvidar que la pedagogía es tanto un saber teórico como práctico.

Igualmente, resulta muy conveniente buscar las posibles formas en que las investigaciones adelantadas en las universidades se acerquen a las instituciones educativas para trabajar en cooperación en la búsqueda de comprensiones sobre el aprendizaje de los profesores y de los estudiantes. Esto implica reconocer a los profesores de educación básica como interlocutores válidos, capaces de participar y hacer aportes en la construcción del conocimiento pedagógico.

\section{Referencias}

Anijovich, R. (2010). La evaluación significativa. Buenos Aires: Paidós.

Bonilla, E. y Rodríguez, P. (2000). Más allá del dilema de los métodos. Bogotá: Editorial Norma.

Caldera, R. y Escalante, D. (2009). Práctica pedagógica de la lectura y formación docente. Revista de Pedagogía, 31(88), 15-37.
Camilloni de, A., Davini, M. C., Edelstein, G., Litwin, E., Souto, M. y Barco, S. (2008). Corrientes didácticas contemporáneas. Buenos Aires: Paidós.

Cordova, D. y Ochoa, K. (2008). Concepciones sobre la enseñanza de la lectura en un grupo de docentes. Revista Investigación y Postgrado, 24(1), 159-187.

Ferreiro, E. y Teberosky, A. (1979). Los sistemas de escritura en el desarrollo del niño. México: Siglo XxI.

Guzmán, R. y Ecima, I. (2011). Conocimiento práctico y conocimiento académico en los profesores del nivel inicial (seis preguntas). Revista Folios, 34, 3-14.

Hernández, D. (2012). Las concepciones de los docentes sobre la escritura en primaria y su enseñanza. Revista Enunciación, 17(1), 40-54.

Jiménez, J. y O’Shanahan, I. (2008). Enseñanza de la lectura: De la teoría y la investigación a la práctica educativa. Revista Iberoamericana de Educación, 45(5), 1-22. Recuperado de: http://www.rieoei.org/ deloslectores/2362JimenezV2.pdf

Litwin, E. (2008). Las configuraciones didácticas. Buenos Aires: Paidós.

Márquez, J. (2008). Lógicas, racionalidades y transformaciones del sentido común. Medellín: Universidad Nacional de Colombia.

Mercado, R. (2002). Los saberes docentes como construcción social. México: Fondo de Cultura Económica.

Rockwell, E. (1995). De huellas, bardas y veredas. La escuela cotidiana. México: Fondo de Cultura Económica.

Tolchinsky, L. (2008). The conditions for literacy learning. Lyon: Literacy workshop.

Unesco (1986). Formación de docentes en las técnicas de lectura y escritura. Recuperado de: http:/unesdoc. unesco.org/images/0006/000687/068739so.pdf

Para citar este artículo

Guzmán, R. (2017). Aprendizaje de los profesores sobre alfabetización y métodos de enseñanza. Folios, (46), 105-116. 